\title{
Pros and cons: Should AIT be considered in all patients with allergic asthma?
}

\author{
Roy Gerth van Wijk ${ }^{1}$ and Ronald Dahl ${ }^{2}$ \\ ${ }^{1}$ Erasmus MC Afdeling Inwendige Geneeskunde \\ ${ }^{2}$ Aarhus Universitet Tandlageskolen
}

July 29, 2021

\begin{abstract}
Allergen immunotherapy (AIT) has gained a permanent place in the therapeutic arsenal for the patient with allergy. Particularly, substantial evidence has been established for the efficacy of AIT in allergic rhinitis. A hallmark of AIT is it disease modifying effect resulting in persistent benefit after the treatment has been terminated. Both the subcutaneous and sublingual mode of administration appear to be safe. It is, however, a matter of debate whether AIT can be implemented for patients with asthma. EAACI and GINA guidelines recommend sublingual AIT in house dust mite driven asthma. The question however remains whether the different available forms of AIT should be used for allergic asthma in general.
\end{abstract}

Pros and cons: Should AIT be considered in all patients with allergic asthma?

Roy Gerth van Wijk ${ }^{1 x}$, Ronald Dahl ${ }^{2}$

Affiliations:

${ }^{1}$ Section of Allergology and Clinical Immunology, department of Internal Medicine. Erasmus Medical Center, Rotterdam. The Netherlands.

${ }^{2}$ Aarhus University, Aarhus, Denmark

${ }^{\mathrm{x}}$ Corresponding author

Roy Gerth van Wijk

Section of Allergology and Clinical Immunology, department of Internal Medicine. Erasmus Medical Center, Rotterdam. The Netherlands.

Keywords: allergy, asthma, allergen immunotherapy, subcutaneous immunotherapy, sublingual immunotherapy

Abstract Allergen immunotherapy (AIT) has gained a permanent place in the therapeutic arsenal for the patient with allergy. Particularly, substantial evidence has been established for the efficacy of AIT in allergic rhinitis. A hallmark of AIT is it disease modifying effect resulting in persistent benefit after the treatment has been terminated. Both the subcutaneous and sublingual mode of administration appear to be safe. It is, however, a matter of debate whether AIT can be implemented for patients with asthma. EAACI and GINA guidelines recommend sublingual AIT in house dust mite driven asthma. The question however remains whether the different available forms of AIT should be used for allergic asthma in general.

PRO (707 words) 
Asthma is a heterogeneous disorder, with many pathophysiological mechanisms underlying the typical clinical presentation. The term "asthma" could be considered as an umbrella diagnosis. Several components are involved in the asthma disease process and "one size" does not fit to all individuals. Each patient should be evaluated for the clinically relevant factors involved, including atopic sensitisation. Provided the diagnosis of asthma is correct, and the specific allergy is present, and a therapeutic extract containing the relevant allergen components is used, then AIT may result in clinical improvement of asthma. The role of AIT in asthma has been addressed in a recent European Academy of Allergy and Clinical Immunology (EAACI) guideline(1) and systematic review(2).

Two important outcomes of AIT were mentioned in the position paper endorsed by The World Health Organization, namely the ability to induce tolerance toward the allergen in question and to change the natural history of the allergic disease(3). Both subcutaneous immunotherapy (SCIT) and sublingual immunotherapy (SLIT) show efficacy and reduce symptom scores and medication use. In addition, SCIT and SLIT improve quality of life, and induce sustained clinical effect after three or more years of treatment. A disease modifying effect is seen based on changes in specific immunologic markers(4).

The persistence of clinical effect also following treatment end is remarkable and not seen for other asthma treatments. The tolerance induced seems to be based on a modification of the immunological processes leading to clinical asthma. This involves a change in $\mathrm{T}$ cell response from an allergic type Th2 dominance to a Th1 dominance. This is reflected by the cytokines produced after allergen contact. Also, an induction of specific regulatory $\mathrm{T}$ cells has been documented. Recently, the role of immunosuppressive regulatory $\mathrm{B}$ cells and IL-10 producing innate lymphoid cells (ILCs) in AIT has been documented (5).

The increase in our understanding of the mechanisms behind AIT (figure 1) paves the way for innovation and further improvement of AIT.

Although there are safety concerns about SCIT, the European Survey on Adverse Systemic Reactions in Allergen Immunotherapy (EASSI) evaluated both SCIT and SLIT, in a total of 4316 patients. Hundred and nine systemic reactions were reported, 97 of them (89\%) concerning SCIT, the most frequent being urticaria, rhinitis, dyspnoea, and cough. Adrenaline was administered in 17 systemic reactions; however, only $65 \%$ of such reactions were classified as anaphylaxis(6). To minimize the risks of systemic reactions, physicians should be experienced and well trained.

The recent EAACI guideline about HDM driven asthma recommends AIT as an add-on to regular asthma therapy for adults with controlled or partially controlled allergic asthma. HDM SCIT is recommended for adults and children, and SLIT drops are recommended for children. Moreover, the Global strategy for asthma (GINA) management and prevention 2021 (8), states that adding SLIT should be considered for adult patients with allergic rhinitis and sensitized to house dust mite with persisting asthma symptoms despite low-medium dose ICS-containing therapy, provided FEV1 is $>70 \%$ predicted.

In clinical trials SLIT for house dust mite allergy was found to reduce moderate/severe exacerbations, improve asthma symptoms and improve quality of life. The treatment also significantly reduced the need for inhaled corticosteroid. This may be related to a concomitant improved lung function, a reduction of the response to allergen provocation(9) and a decrease of eosinophilic bronchial inflammation.

In conclusion (figure 1), properly diagnosed and well-selected patients with allergic asthma may benefit from AIT, provided that patients are treated with well characterized and standardized extracts containing the relevant allergens. AIT is the only treatment that may have a long term immune modulating effect in asthma. In clinical terms, this could be seen by a persistence of clinical improvement several years after termination of AIT. The choice for a specific AIT product should always be based on the availability of high quality efficacy and safety data.

\section{PRO}

- AIT with HDM tablets reduces the risks of exacerbations,

- AIT with HDM tablets reduces the amount of ICS needed for asthma control, 
- AIT is the only disease modifying treatment resulting in a persistent reduction of symptoms,

- AIT is a safe treatment with few severe side effects in SCIT and transient mild side effects in SLIT,

- The increasing understanding of the immunological mechanisms behind AIT paves the way for innovative and improved AIT.

CON (669 words)

In 110 years allergen immunotherapy has evolved as one of the therapeutic pillars for allergic patients with rhinoconjunctivitis. The question has to be addressed whether this is also true for the treatment of allergic asthma. The EAACI guideline for house dust mite-induced (HDM) asthma recommends SCIT for adults and children(1). However, using the GRADE approach the authors consider the evidence of low quality. A significant heterogeneity of HDM SCIT studies was observed. Moreover, due to lack of evidence no recommendation could be provided for the use of HDM SCIT to decrease exacerbations, improve asthma control and lung function, or to decrease nonspecific airway hyperresponsiveness. SLIT drops were only recommended for children, but again based on studies with low-quality evidence (1). At present, SLIT drops are not on the market in Europe and the US. Based on a large RCT from Virchow, HDM sublingual tablets are recommended for adults to prevent asthma exacerbations(7). This RCT can be considered as a landmark study. There are, however, no data yet supporting efficacy of HDM tablets in children. SLIT tablets for children are under investigation.

The recent EAACI systematic review focusing on different allergens demonstrated efficacy of AIT in general with reductions in short-term symptom and medication scores, whereas a subgroup-analysis confirmed a beneficial efficacy for SCIT but questionable effects for SLIT(2). It was however, stated that these findings needed to be interpreted with caution as the majority of trials are of high or uncertain risk of bias. The questionable effect for SLIT confirms an earlier Cochrane study which was hampered by lack of data for important outcomes such as exacerbations and quality of life and by use of different non-validated symptom and medication scores(10)

In this review, few studies were focused on studies were focused on asthma caused by pet and fungal allergy. Efficacy for cat and dog allergy was demonstrated, while efficacy for fungal asthma was suggested but not confirmed(2). Moreover, the animal allergy subgroup analysis was mainly based on older small SCIT studies conducted several decades ago.

AIT aims to change the natural course of the disease. Despite observational studies suggesting long-term efficacy, randomised long-term studies have not yet been conducted in asthmatics, neither with SCIT nor with $\operatorname{SLIT}(1)$.

Cost-effectiveness was also addressed in the EAACI guidelines(11). Although cost-effectiveness over standard treatment was suggested, all studies were of low methodological quality. One SCIT and two SLIT studies only satisfied the eligibility criteria. One study only focused on asthma without rhinitis. Cost-effectiveness could not be calculated as no attempt was made to convert outcome measures in a general quality of life measure such as quality of life adjusted life years (QALYS). The second study calculated QALYS but did not characterise the uncertainty of the estimate. In addition, the third study did not measure QALYS(11).

Finally, some other caveats. Clinicians should be aware that sensitization to an allergen by itself does not justify AIT. The allergen should be considered clinically as a major trigger. Moreover, AIT is demanding much from both patients and health care providers. The long duration of treatment and the slow onset of treatment effects may compromise adherence to treatment.

In conclusion (figure 1), although good results have been obtained with HDM sublingual tablets in adult asthmatic patients, the evidence for AIT in asthmatic children is weak. Moreover, more studies are needed to assess long-term efficacy, to evaluate AIT with other allergens, particularly animal allergens and fungi. High-quality cost-effectiveness studies are needed to meet the requirements of national health care systems.

CON 
- Robust effects have been demonstrated with HDM tablets in adults only, but evidence for other routes (SCIT) and methods (SLIT drops) of administration is weak,

- Evidence for the efficacy of AIT with other allergens, such as animal allergens and fungi is limited, There is an urgent need to establish solid evidence for AIT in asthmatic children,

- Good data on sustained or long-term efficacy for AIT in asthma are not available yet,

- The number of cost-effectiveness studies is low. The methodological quality of available studies is limited.

\section{CONFLICT OF INTEREST}

Both authors declare no conflict of interest.

\section{Hosted file}

image1.emf available at https://authorea.com/users/428287/articles/532179-pros-and-consshould-ait-be-considered-in-all-patients-with-allergic-asthma

Figure 1 AIT in asthma: benefits, research gaps and immunological mechanisms

1. Agache I, Lau S, Akdis CA, Smolinska S, Bonini M, Cavkaytar O, et al. EAACI Guidelines on Allergen Immunotherapy: House dust mite-driven allergic asthma. Allergy. 2019;74(5):855-73.

2. Dhami S, Kakourou A, Asamoah F, Agache I, Lau S, Jutel M, et al. Allergen immunotherapy for allergic asthma: A systematic review and meta-analysis. Allergy. 2017;72(12):1825-48.

3. Bousquet J, Lockey R, Malling HJ. Allergen immunotherapy: therapeutic vaccines for allergic diseases. A WHO position paper. J Allergy Clin Immunol. 1998;102(4 Pt 1):558-62.

4. Ozdemir C, Kucuksezer UC, Akdis M, Akdis CA. Mechanisms of Aeroallergen Immunotherapy: Subcutaneous Immunotherapy and Sublingual Immunotherapy. Immunol Allergy Clin North Am. 2016;36(1):71-86.

5. Komlosi ZI, Kovacs N, Sokolowska M, van de Veen W, Akdis M, Akdis CA. Mechanisms of Subcutaneous and Sublingual Aeroallergen Immunotherapy: What Is New? Immunol Allergy Clin North Am. 2020;40(1):114.

6. Calderon MA, Vidal C, Rodriguez Del Rio P, Just J, Pfaar O, Tabar AI, et al. European Survey on Adverse Systemic Reactions in Allergen Immunotherapy (EASSI): a real-life clinical assessment. Allergy. 2017;72(3):462-72.

7. Virchow JC, Backer V, Kuna P, Prieto L, Nolte H, Villesen HH, et al. Efficacy of a House Dust Mite Sublingual Allergen Immunotherapy Tablet in Adults With Allergic Asthma: A Randomized Clinical Trial. JAMA. 2016;315(16):1715-25.

8. Global Initiative for Asthma. Global strategy for asthma management and prevention 2020 [Available from: www.ginasthma.org. Accessed 30 June 2021)

9. Rosewich M, Girod K, Zielen S, Schubert R, Schulze J. Induction of Bronchial Tolerance After 1 Cycle of Monophosphoryl-A-Adjuvanted Specific Immunotherapy in Children With Grass Pollen Allergies. Allergy Asthma Immunol Res. 2016;8(3):257-63.

10. Fortescue R, Kew KM, Leung MST. Sublingual immunotherapy for asthma. Cochrane Database Syst Rev. 2020;9:CD011293.

11. Asaria M, Dhami S, van Ree R, Gerth van Wijk R, Muraro A, Roberts G, et al. Health economic analysis of allergen immunotherapy for the management of allergic rhinitis, asthma, food allergy and venom allergy: A systematic overview. Allergy. 2018;73(2):269-83. 


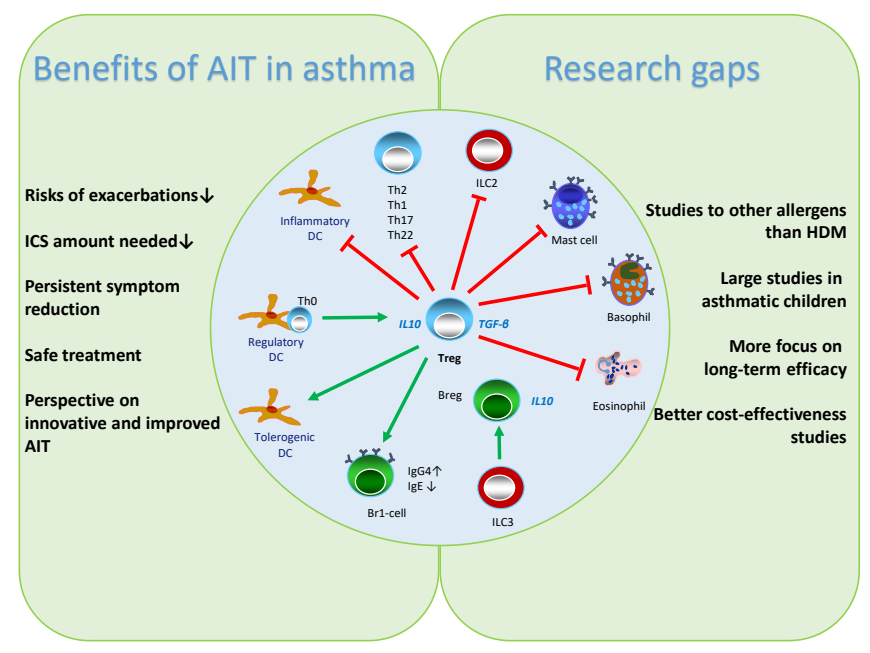

